\title{
Ultra-high-sensitivity color imaging via a transparent diffractive-filter array and computational optics: supplementary material
}

\author{
Peng Wang and Rajesh MenON ${ }^{*}$ \\ Department of Electrical and Computer Engineering, University of Utah, Salt Lake City, UT 84112 \\ *Corresponding author: rmenon@eng.utah.edu \\ Published 29 October 2015
}

This document provides supplementary information to "Ultra-high-sensitivity color imaging via a transparent diffractive-filter array and computational optics," http://dx.doi.org/10.1364/0PTICA.2.000933. It contains modeling and mathematical formulations for far-field scalar diffraction simulation of the proposed diffractive filter array (DFA). The patterning technique based on gray-scale lithography is described. Both schematics and photographs of the DFA-sensor assembly and the experimental setups are shown. In this document, several critical mechanical alignment methods and spectrum reconstruction algorithms (iterative and non-iterative approaches) are depicted in detail. Here, we also further extend some of our discussions in the manuscript, including cross-talk effect and tolerance analysis in depth-of-focus, noise and acceptance angle. The numerical methods to evaluate the performance of DFA with sensor pixels as small as $\mathbf{1 . 6 7} \boldsymbol{\mu m}$ are also included. (C) 2015 Optical Society of America

http://dx.doi.org/10.1364/optica.2.000933.s001

1. Device simulation model

The generalized geometry of the proposed imaging system comprised of the diffractive-filter array (DFA) and the monochrome sensor array is schematically shown in Fig.S1. The DFA (blue grid) is discretized into square pixels with uniform sizes $(\Delta x=\Delta y)$. The image space (red grid) is defined in the same grid size. Here, $\Delta x$ and $\Delta y$ are grid sizes used in simulation. The square sizes of the DFA device and the sensor array are assumed integer multiples of this simulation grid size.

The simulation has grid size $\Delta x=\Delta y=1 \mu \mathrm{m}$. The DFA has square size $\Delta \times \Delta=3 \mu \mathrm{m} \times 3 \mu \mathrm{m}$, occupying $3 \times 3$ simulation grids. The sensor has pixel size $\Delta^{\prime} \times \Delta^{\prime}=6 \mu \mathrm{m} \times 6 \mu \mathrm{m}$. The DFA topography can be expressed by:

$$
h(x, y)=\sum_{m} \sum_{n} \Delta h \cdot p_{(m, n)} \cdot \operatorname{rect}\left(\frac{x-m \Delta}{\Delta}\right) \cdot \operatorname{rect}\left(\frac{y-n \Delta}{\Delta}\right)
$$

in which $\Delta h=H /\left(N_{\text {levels }}+1\right)$ is the unit height, $H$ is the maximum height and $N_{\text {levels }}$ is the total number of quantization levels; $p(m, n)$ is the a positive integer within the interval $\left[0, N_{\text {levels }}\right]$; $\operatorname{rect}()$ is the rectangle function with integer indices $m$ and $n$. In practice, the DFA height profile is generated by a function in Matlab - 'rand'. It essentially generates a matrix of random numbers.
Since the DFA is relatively thin and the imaginary part of the refractive index of the material (Shipley 1813 positive-tone photoresist) is negligibly small, we can reasonably ignore its absorption. Therefore, the transmission function is given by:

$$
\begin{aligned}
& T(x, y)=e^{i \varphi(x, y)} \\
& =1+\sum_{m} \sum_{n} \operatorname{rect}\left(\frac{x-m \Delta}{\Delta}\right) \cdot \operatorname{rect}\left(\frac{y-n \Delta}{\Delta}\right) \cdot\left(e^{i a \cdot p_{(m, n)}}-1\right)
\end{aligned}
$$

where $\alpha=k \cdot \Delta h \cdot(n-1)$, and $k=2 \pi / \lambda$ is the wave number and $n$ is the real part of the refractive index of Shipley 1813. This assumes patterning the DFA on a clear, non-absorbing substrate such as glass.

The diffracted light field at the image plane $\left(X^{\prime}-Y^{\prime}\right.$ coordinate system) with distance $Z=d$ is described by the Fresnel transformation [1,2]:

$$
\begin{aligned}
& U\left(x^{\prime}, y^{\prime}, d\right) \\
& =\frac{e^{i k d}}{i \lambda d} \cdot \iint g_{\text {illum }}(x, y) \cdot T(x, y) \cdot e^{i \frac{k}{2 d}\left[\left(x^{\prime}-x\right)^{2}+\left(y^{\prime}-y\right)^{2}\right]} d x d y
\end{aligned}
$$

in which $K_{(m, n)}=e^{i a \cdot p_{(m, n)}}-1$. An on-axis unit-amplitude illumination light was assumed $g_{(m, n)}=1$. Either periodic boundary condition or finite-aperture boundary condition can be 
applied to the illuminating light field for the following numerical analysis.

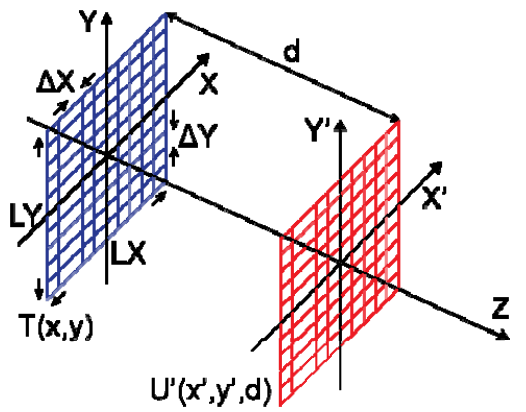

Fig. S1. Schematic illustration of the generalized simulation model of the proposed color imaging design.

\section{Device fabrication}

In gray-scale lithography, the write head scans through the sample surface and the exposure dose at each point is modulated with different gray-scales [3,4]. Most photoresist is characterized by a contrast curve [5]. Different depths in accord with different exposure doses are achieved after development. Greater dose leads to deeper feature. Before patterning structures, it is needed to calibrate this contrast curve. Figure S2(a) gives a typical calibration plot, based upon which the exposure gray-scale at each point is given. The surface roughness measurement (max-min height $\sim 2.8 \mathrm{~nm}$ ) by AFM demonstrates the excellent smoothness of surface after gray-scale fabrication, which suppresses undesired scattering.
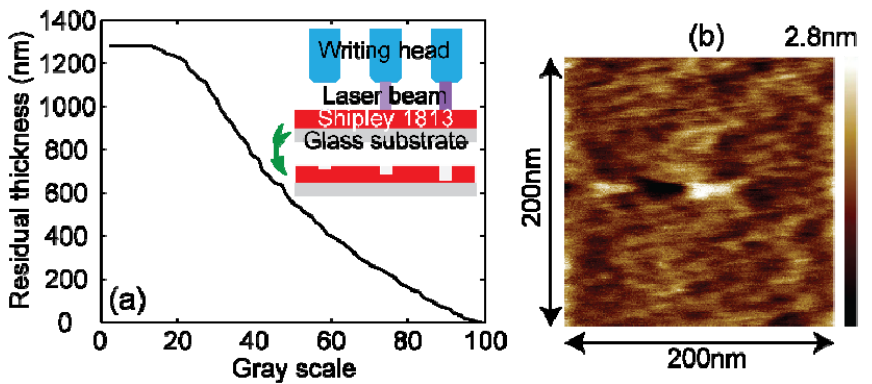

Fig. S2. (a) Calibration curve of Shipley 1813 photoresist at laser power of $12 \mathrm{~mW}$ and $40 \%$ duration factor. Inset: schematic of grayscale lithography on a positive photoresist. (b) Surface roughness measurement of the fabricated DFA by AFM.

The fabrication procedure:

1) RCA clean a 2-inch diameter fused silica wafer.

2) Spin coat HMDS on the fused silica wafer, 45sec @ 6000rpm. Leave it in the fume hood for 10min for sufficient evaporation.

3) Spin coat Shipley 1813 photoresist (positive), 45sec @ 4000rpm with ramp rate $1000 \mathrm{rpm} / \mathrm{s}$.

4) Soft bake on a hotplate, $90 \mathrm{sec} @ 115^{\circ} \mathrm{C}$.

5) Dehydration in fume hood for $1 \mathrm{hr}$.

6) Gray-scale exposure by the Heidelberg microPG101 with $3 \mu \mathrm{m}$ mode. Power: $12 \mathrm{~mW}$; duration factor: $40 \%$; mode: unidirectional; scan-scale: $2.74 \mathrm{MHz}$.

7) Development in AZ MIF 300 developer for $1 \mathrm{~min}$.

8) Rinse in DI water for $1 \mathrm{~min}$. Dry the sample by blowing Nitrogen.

\section{DFA-sensor assembly}

The schematic of the DFA-sensor assembly is illustrated in Fig. S3 with every part and critical dimension labelled in detail. Based on the measured and specified [6] dimensions (Fig. S3), we are able to determine the gap $g$ between the front surfaces of optical mounts from the desired image distance $d$. Three distances $d=0.3 \mathrm{~mm}, 0.5 \mathrm{mmm}$ and $1.5 \mathrm{~mm}$ are tested, resulting in $g=2.67 \mathrm{~mm}$, $2.87 \mathrm{~mm}$ and $3.87 \mathrm{~mm}$, respectively. Note the custom-designed camera holder is machined out of a 2"-diameter Teflon rod. The camera board, together with the sensor, is fixed upon the holder by M2 screws. The transparent cover film on top of the sensor is measured about $0.10 \mathrm{~mm}$ thick.
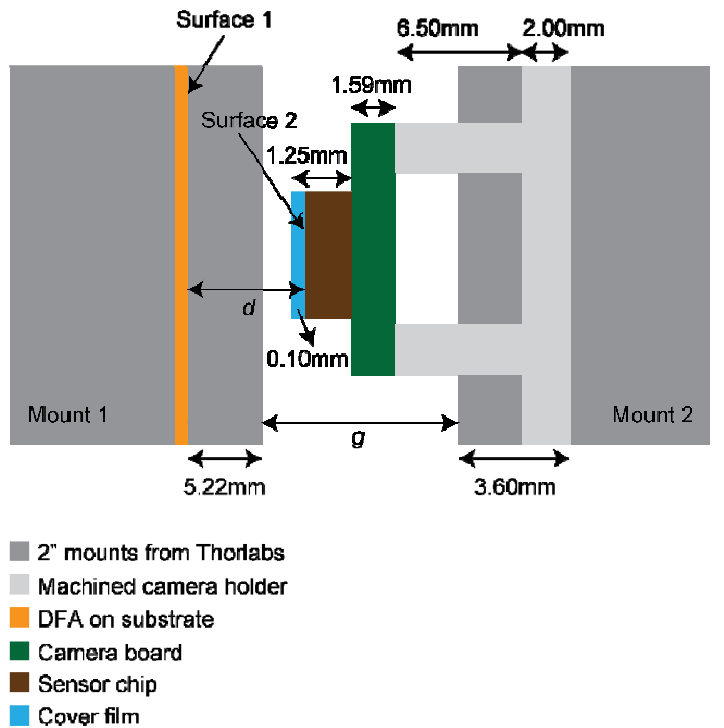

Fig. S3. Configuration of the DFA-sensor assembly with labelled dimensions.

\section{Setups}

\subsection{SS-PSF calibration setup}

In SS-PSF calibration setup, the DFA is fixed on a mount that is able to rotate in XY plane with high resolution [6]. The fiber tip is at $d$ away from the DFA surface and is mounted on a XY 2-axis automated stage.

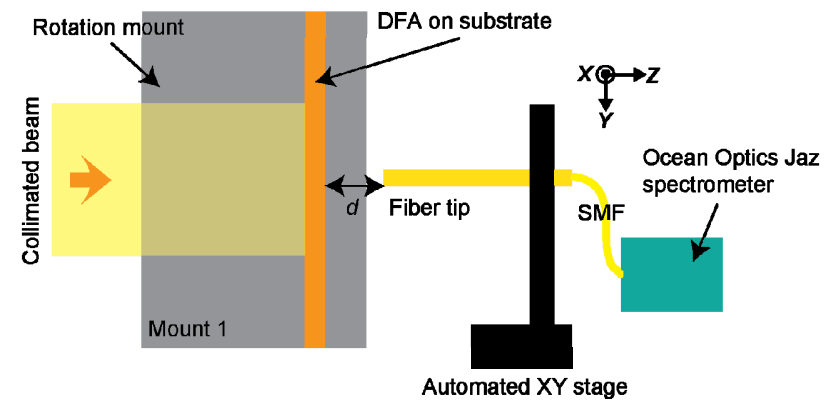

Fig. S4. Schematic of the SS-PSF calibration setup.

\subsection{Color imaging setup}

In color imaging experiment, the DFA and the sensor is assembled according to Fig. S3. The sample is firstly color-printed on a piece of transparency. Then it is tightly glued upon a cleaned glass substrate. This is fixed on a cage mount and placed in proximity $(\sim 50 \mu \mathrm{m})$ to the back side of the DFA substrate. The mount is controlled by a micrometer stage. 

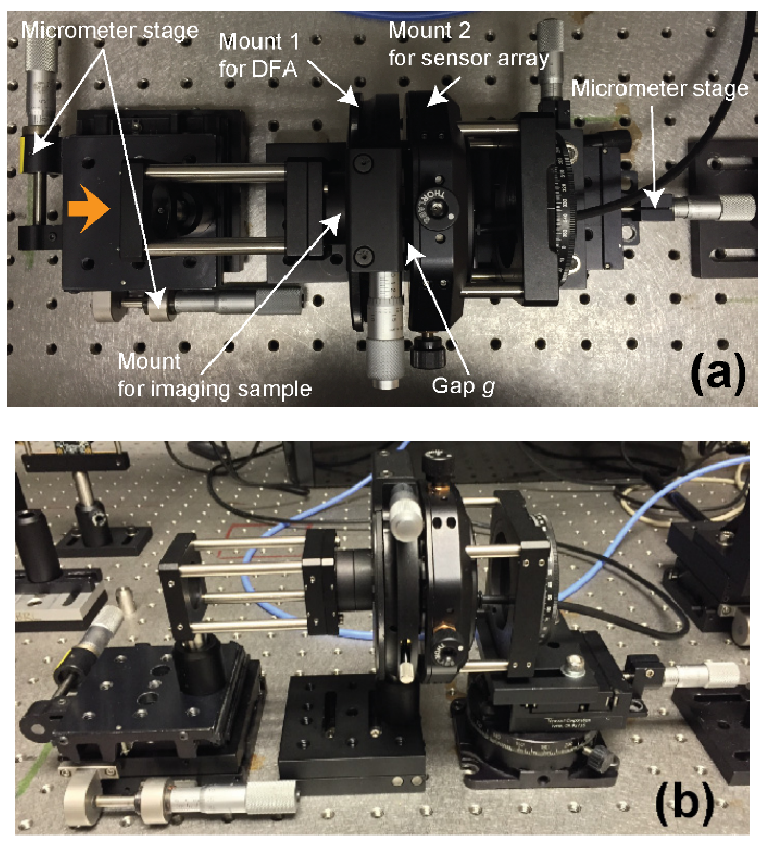

Fig. S5. Photographs of the color imaging setup. The expanded and collimated beam from a super-continuum source illuminates from the left side. (a) Top view. (b) Side view.

\subsection{Angular response measurement setup}

In the angular response measurement setup, lens 2, mounts 1 and 2 are fixed on a two-axis micrometer stage. As illustrated in Fig. S6(a), lens 1 firstly focuses the collimated beam, then off-axis illumination is created by moving lens 2, together with the DFAsensor assembly, at XY plane. Incident angles in two orthogonal coordinates are computed by $\theta x=\tan ^{-1}(\Delta x / d)$ and $\theta y=\tan ^{-1}(\Delta y / d)$, in which $\Delta x$ and $\Delta y$ are the movements relative to the central reference point. Note that now the gap $g$ between two mounts is controlled by a $0.01 \mathrm{~mm}$-resolution caliper. Collimated uniform beam is incident on lens 1 .
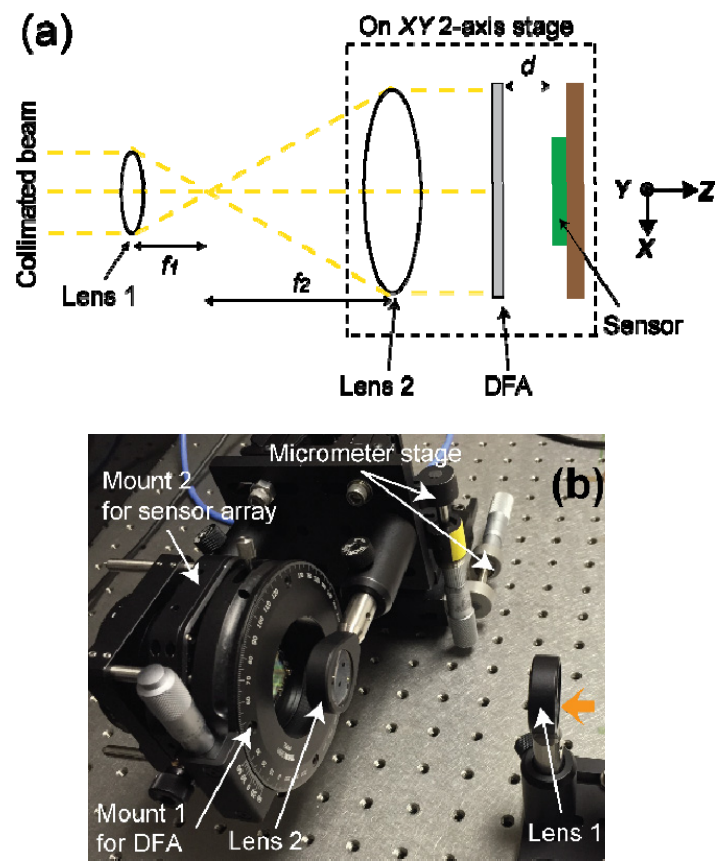

Fig. S6. Schematic illustration (a) and photograph (b) of the angular response measurement setup.

\section{Alignment techniques}

\subsection{Gap control in SS-PSF calibration}

To make the gap between the SMF fiber-tip and the DFA surface accurately, the following method is applied. Figure S7 also explains the process.

a) Place two single-side-polished 2" silicon wafers in mount 1. Both polished sides face outward. Their back sides are in tight contact.

b) Adjust the mount 1 to make the wafers perpendicular to light path by observing the reflected light (from wafer 1) on the optic component (a lens on its mount).

c) Move the fiber tip slowly towards wafer 2 by a micrometer stage and observe the reflected light (from wafer 1) at the same time.

d) Write down the micrometer reading when the fiber-tip just touches the surface of wafer 2 . This marks $d=0$ reference point. Now the reflected light just becomes slightly larger than before due to slight deflection of wafer. (a)

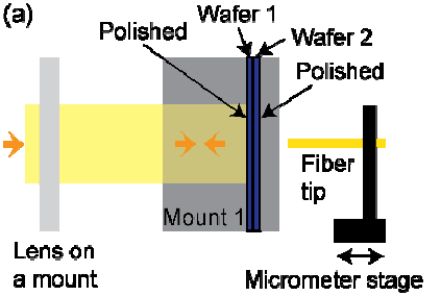

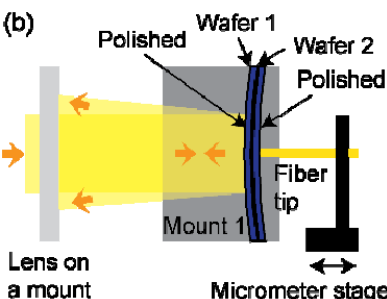

Fig. S7. Schematic explaining gap control method for SS-PSF calibration. (a) Before the fiber tip touches the polished side of wafer 2. (b) Right after the fiber tip touches the polished side of wafer 2.

\subsection{Angle alignment in SS-PSF calibration}

In SS-PSF calibration, it is important to align the scan direction of fiber tip with the patterned DFA.

a) Mount the DFA on a 2" rotation mount with fine angle resolution (mount 1). Fix fiber tip at some distance, connected to an Ocean Optics Jaz spectrometer.

b) Measure spectrum.

c) Move fiber tip a certain distance away in either $\mathrm{X}$ or $\mathrm{Y}$ direction by the automated $\mathrm{XY}$ stage. Here, it is moved along $\mathrm{X}$ for $180 \mu \mathrm{m}$ (or $10 \mathrm{DFA}$ periods equivalently). Measure spectrum again at this new position.

d) Repeat b) and c) at various rotation angles using mount 1 .

e) Calculate the difference between the two spectrum measurements at two positions within a range of angles.

f) Pick the angle that gives the minimum difference. Now the scan direction is aligned with the DFA.

Note the mount has rotation resolution of $0.028^{\circ}$, which is sufficient for current application. For Fig. S8, in a coarse measurement, the angle resolution is $0.14^{\circ}$ (blue curve), while in a finer measurement, the resolution is $0.028^{\circ}$ (black curve). 


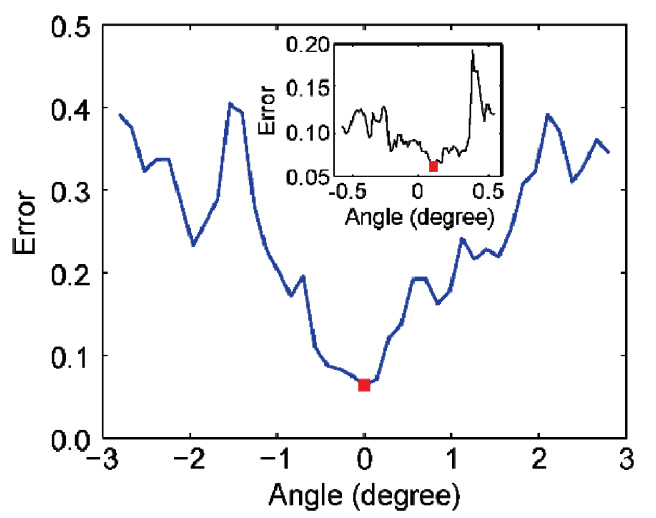

Fig. S8. Result of angle alignment for SS-PSF calibration with low angle resolution (blue) and high resolution (inset, black). The optimal angle is labelled by red square.

\subsection{Gap control in color imaging}

a) Compute the gap $g$ between surfaces of two optical mounts based on the target $d$ and the schematic in Fig. S3.

b) Mount DFA on mount 1 (the high-precision rotation mount). Adjust the angle in XZ plane by observing the image reflected from the back side of the DFA substrate so that the DFA is perpendicular to the light path.

c) Move mount 2, controlled by a micrometer stage, in contact with mount 1 . Now the camera and its holder are not mounted. Write down the micrometer stage reading $p$. Mounts 1 and 2 are in parallel.

d) Mount the camera and its holder onto mount 2. Move mount 2 to the position with the micrometer stage reading $p+g$. Now the sensor is at distance $d$ from the DFA.

\subsection{Angle alignment in color imaging}

The orientation of DFA has to be aligned with the sensor pixels. a) Assemble DFA and sensor based on Fig. S3. Build the setup based on Fig. S6. Take image of just the uniform illumination beam by the monochrome camera.

b) Rotate mount 1 to a new angle. Take a new image.

c) Repeat b) for many angles.

d) Compute the differences between single-DFA-period images $(3 \times 3$ sensor pixels) at two ends of one row of the entire image captured at different rotation angles. The angle that gives the minimum difference marks the optimal angle for imaging. Now the DFA and the sensor array are aligned accurately.

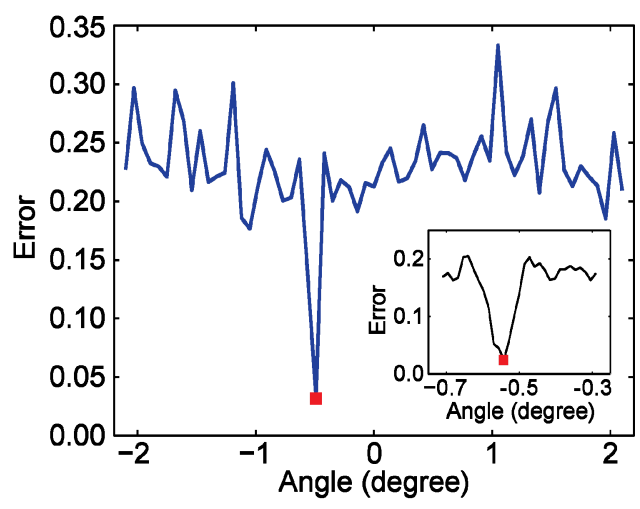

Fig. S9. Result of angle alignment between the DFA and the sensor array for color imaging with low angle resolution (blue) and high resolution (inset, black). The optimal angle is labelled by red square.
5.5 Numerical alignment between calibration data and sensor array

Since there is inevitably lateral offset between the calibrated SSPSF data and the image captured by the monochrome sensor array, it is needed to align the PSF images with the sensor array. This is achieved by numerical alignment. In this method, for a certain known spectrum, different combinations of lateral shifts (X and Y directions) are applied to the PSF images and the camera image is numerically synthesized. This is compared to the real camera image. The combination ( $\mathrm{X}$ and $\mathrm{Y}$ shifts) that gives the best fit (the minimum difference) is considered as the optimal one and used for all the imaging experiments. Figure S10 shows the numerical alignment results. An $X=13 \mu \mathrm{m}$ shift together with $\mathrm{Y}=9 \mu \mathrm{m}$ shift offers the best fit.

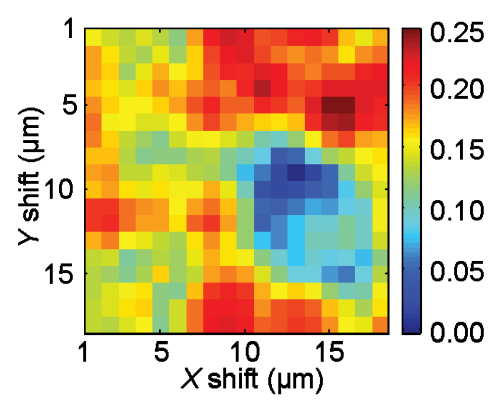

Fig. S10. Result of numerical alignment (lateral shifts in $\mathrm{X}$ and $\mathrm{Y}$ directions) between DFA and sensor array.

\section{Color error tolerance}

In order to evaluate the performance of color reconstruction, it is fundamental to understand how much color error is tolerable. Figure S11 shows a simple color error tolerance test. Different computer-generated random color errors in percentage of 256 levels (8-bit) are applied to three basic colors (blue, green and red). Based on visual inspection, it seems that generally color errors below $10 \%$ are acceptable while errors beyond $10 \%$ gives evident discrepancies.

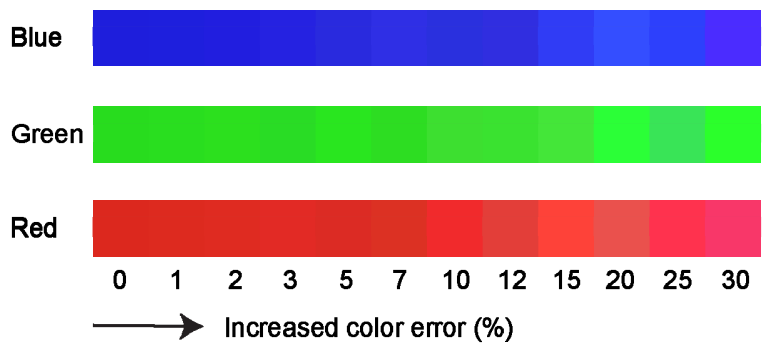

Fig. S11. Color error tolerance test by adding various random color errors in percentage to three basic colors.

\section{Spectrum reconstruction algorithms}

\subsection{Direct-binary-search}

Direct-binary-search is an iterative algorithm [7-10]. Its figureof-merit is the residual norm $\|\mathbf{P S F} \cdot \mathbf{\Psi}-\mathbf{S}\|_{2}$. At first, an initial guess of compound photon flux spectrum $\Psi$ is given. Usually, a random spectrum is used. During one iteration, all wavelength samples within this spectrum is perturbed in a pre-designed manner (random as well). A positive unit perturbation is tried. If this residual norm is reduced, then this perturbation is kept, otherwise 
a negative unit perturbation is applied to this wavelength. If the residual norm is reduced, then this negative perturbation is kept, otherwise it proceeds to the next wavelength sample. The guessed spectrum $\boldsymbol{\Psi}$ is updated accordingly. One iteration stops when all wavelength samples are traversed. Termination conditions guarantee convergence, such as a maximum number of total iterations or a minimum residual norm improvement between two iterations. [11]

The three basic parameters defining the DBS algorithm process in this work are: wavelength sampling rate $7 \mathrm{~nm}$, unit perturbation 0.005 , initial solution Gaussian function centered at $550 \mathrm{~nm}$ with FWHM 100nm. Note that they are already optimized for spectrum reconstruction. The spectrum $\boldsymbol{\Psi}$ is normalized. 200 to 300 iterations are usually required.

\subsection{Regularization}

Based on the main text, it is essentially an inverse problem to solve $\boldsymbol{\Psi}$ from $\mathbf{S}$ [12]. However, matrix PSF has usually large condition number, it is mathematically implausible to obtain its inverse. Thus regularization is used [13]. Firstly, the system matrix PSF is decomposed by singular-value decomposition (SVD): $\mathbf{P S F}=\mathbf{U} \boldsymbol{\Sigma} \mathbf{V} . \boldsymbol{\Sigma}$ is a diagonal matrix with the singular values as its diagonal elements, arranged in a descending manner $\left(\sigma_{1} \geq \sigma_{2} \geq \sigma_{3} \geq \ldots \geq \sigma_{\mathrm{m}}\right)$. The columns of $\mathbf{U}$ and $\mathbf{V}$ matrices $\left(\mathrm{u}_{1}, \mathrm{u}_{2} \ldots \mathrm{um}_{\mathrm{m}}\right.$, and $\mathrm{v}_{1}, \mathrm{v}_{2} \ldots \mathrm{v}_{\mathrm{m}}$ ) contain the $\mathrm{m} \times 1$ left and right singular vectors in spatial and spectral domains, respectively.

The regularization technique stabilizes the problem by minimizing both the residual norm $\|$ PSF. $\Psi-\mathbf{S} \|_{2}$ and the solution norm $\|\mathbf{S}\|_{2}[12,13]$. Usually, a regularization parameter $\omega$ is selected to balance these two terms. For a commonly-used Tikhonov regularization, the goal is stated as [13]:

$$
\min \left\{\|\mathbf{A x}-\mathbf{b}\|_{2}^{2}+\omega^{2}\|\mathbf{x}\|_{2}^{2}\right\}
$$

It can be equally formulated as applying filter factors to solution vectors, from the computational perspective [13]:

$$
\mathbf{x}_{\omega}=\sum_{i=1}^{n} \phi_{i}^{[\omega]} \frac{u_{i}^{T} \mathbf{b}}{\sigma_{i}} v_{i},
$$

in which the filter factor is defined by [13]:

$$
\phi_{i}^{[\omega]}=\frac{\sigma_{i}^{2}}{\sigma_{i}^{2}+\omega^{2}} .
$$

In Eq.(S5), we call $\mathbf{x}_{\omega}$ the Tikhonov regularization solution with parameter $\omega$. The parameter $\omega$ is selected based on the widelyknown L-curve technique $[12,13]$. Much too small $\omega$ leads to results overwhelmed by noises, while much too large $\omega$ leads to over-smoothed distorted results [12]. Note that in order to make the regularization algorithm work, the system matrix PSF has to be a square matrix. Here, there are nine sensor pixels $(3 \times 3)$, therefore we take nine equally-spaced spectral samples: $470 \mathrm{~nm}, 497 \mathrm{~nm}$, $524 \mathrm{~nm}, 551 \mathrm{~nm}, 578 \mathrm{~nm}, 605 \mathrm{~nm}, 632 \mathrm{~nm}, 659 \mathrm{~nm}$ and $686 \mathrm{~nm}$.

\section{Cross-talk effect}

Periodic boundary condition is assumed in the DFA device. Each repeating unit consists of $6 \times 63 \mu \mathrm{m}$-pixels. Due to higher diffraction orders, light transmits through one unit (or period) can affect its neighboring units. Basically, light spreads out. Therefore, image from one unit represents actually the combined contributions from some of its neighboring units. Unless the color remains unchanged over a large number of units, the reconstructed color is compromised in accuracy. This is a cross-talk effect. It also dictates the ultimate spatial resolution. To evaluate this effect, we simulate diffraction patterns of one single DFA unit at different propagation distances. According to diffraction theory, light with the longest wavelength possesses the greatest diffraction angle, therefore $\lambda=700 \mathrm{~nm}$ is considered in simulations. Figure S12 summarizes the simulated diffraction patterns. For $d=0.3 \mathrm{~mm}$, the diffraction pattern is dominantly constraint within a region of about $90 \mu \mathrm{m} \times 90 \mu \mathrm{m}$. For $d=0.5 \mathrm{~mm}$ and $1.5 \mathrm{~mm}$, the light energy is confined within around $150 \mu \mathrm{m} \times 150 \mu \mathrm{m}$ and $450 \mu \mathrm{m} \times 450 \mu \mathrm{m}$ regions.

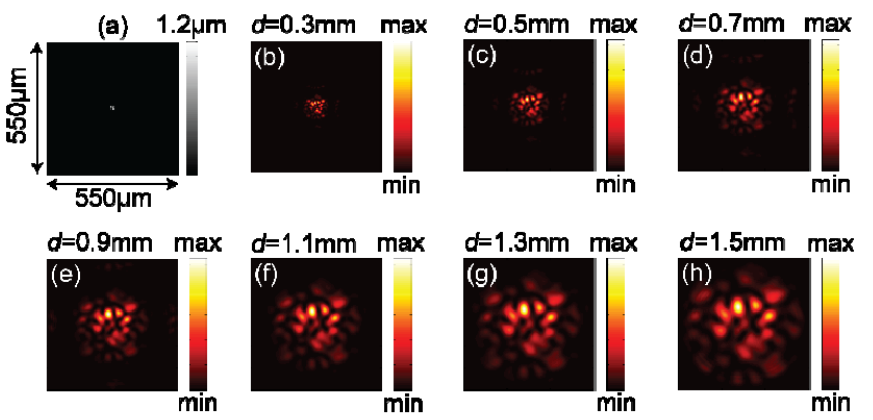

Fig. S12. (a) Original design height distribution $(18 \mu \mathrm{m} \times 18 \mu \mathrm{m})$. (b)-(h) Simulated diffraction patterns of one single DFA unit at various distances at $\lambda=700 \mathrm{~nm}$.

To provide a rough estimate on spatial resolution, it is interesting to conduct a test similar to Abbe's resolution criterion (Fig. S13). Two diffraction patterns are placed next to each other at different displacements. To be consistent with our experiments, we assume the light source coherent. Take $d=0.3 \mathrm{~mm}$ for example. When displacement is below $90 \mu \mathrm{m}$, the two pattern interact with each other and it is difficult to distinguish them apart. The interference fringes at the boundary are very clear. When displacement grows beyond $90 \mu \mathrm{m}$, there is negligible interactions. This critical displacement is taken as the available spatial resolution.

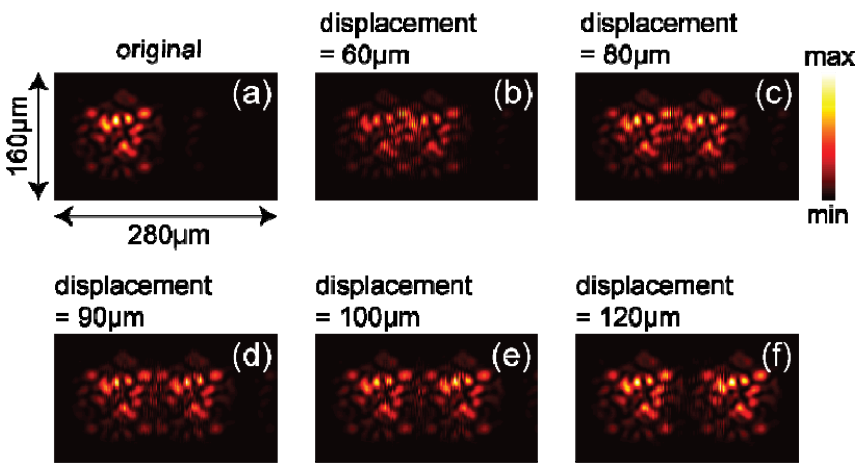

Fig. S13. (a) Original diffraction pattern at $d=0.3 \mathrm{~mm}$ and $\lambda=700 \mathrm{~nm}$. (b)-(f) Numerically synthesized diffraction patterns of two identical patterns separated by different displacements. $90 \mu \mathrm{m}$ is the critical displacement.

\section{Tolerance analysis}

\subsection{Depth-of-focus}

As discussed in the alignment section, it is critical to make sure gap $d$ between the DFA and the sensor is as close to the nominal 
value (used in SS-PSF calibration as well) as possible. Therefore, it is instructive to understand the depth-of-focus of the DFA-sensor camera. We simulated color error (in \% of the 256 levels) of the imaging system at various defocus locations. They are plotted in Fig. S14. Applying a color error of $10 \%$ as the criterion, we can obtain depth-of-focus $\pm 12 \mu \mathrm{m}, \pm 30 \mu \mathrm{m}$ and $\pm 60 \mu \mathrm{m}$ for three nominal gaps $d=0.3 \mathrm{~mm}, 0.5 \mathrm{~mm}$ and $1.5 \mathrm{~mm}$, respectively. These are well within the movement resolution of commonly used micrometer stage (step size $10 \mu \mathrm{m}$ ). Therefore, it is possible to control the gap between the DFA and the sensor with reasonable accuracy and thus achieve acceptable color reconstruction fidelity. The depth-of-focus is concluded to be linearly proportional to the gap $d$.

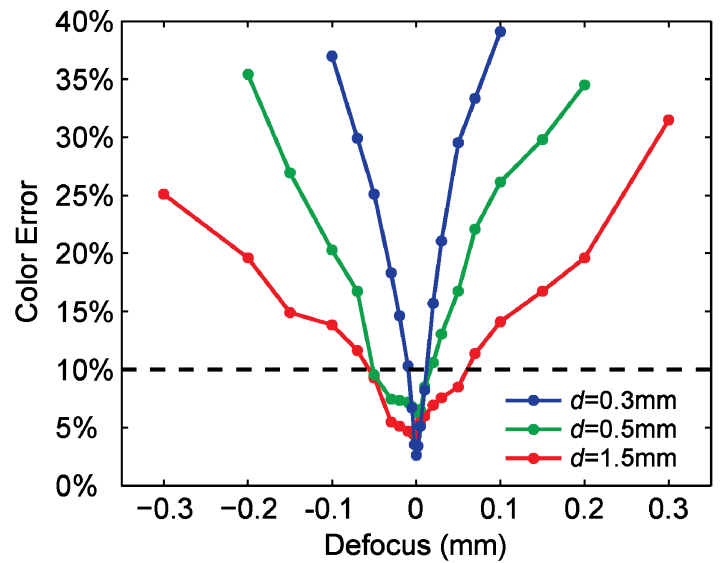

Fig. S14. Plots of color errors in percentage versus defocus locations for three nominal gaps $d=0.3 \mathrm{~mm}$ (blue line), $0.5 \mathrm{~mm}$ (green line) and $1.5 \mathrm{~mm}$ (red line). The 10\% color error tolerance threshold (black dashed line) is used.

\subsection{Acceptance angle}

Acceptance angle is important for diffraction devices since offaxis incidence imparts a phase shift to the wave-front that leads to a lateral shift of the diffraction pattern. Our calibration experiments assumed strictly normal incidence. An optical setup is devised to measure angular response (see Fig. S6). An angular response function is defined by:

$$
\begin{aligned}
& R(\theta x, \theta y) \\
& =1-\text { normalize }\left(\sum_{x} \sum_{y}|I(\theta x, \theta y, x, y)-I(0,0, x, y)|\right)
\end{aligned}
$$

Here, $I(\theta x, \theta y, x, y)$ and $I(0,0, x, y)$ are the images at angle $(\theta x, \theta y)$ and normal incidence. Note that no sample is used. The second term in Eq. (S7) is the normalization over a set of angles $(\theta x, \theta y)$. As shown in Figs. S15(a)-(c), $R(\theta x, \theta y)$ drops with larger angles. This makes sense because as incoming light is more and more off normal, the image turns less and less similar to the one at normal incidence. By considering the angles at $R(\theta x, \theta y)=0.5$ as the acceptance angle $\theta a$, Figs. S15(a)-(c) indicate that $\theta a=0.19^{\circ}, 0.13^{\circ}$ and $0.05^{\circ}$ for $d=0.3 \mathrm{~mm}, 0.5 \mathrm{~mm}$ and $1.5 \mathrm{~mm}$, respectively. They are all roughly equivalent to relative lateral shift of $\Delta x \sim 1 \mu \mathrm{m}$. To guarantee the camera's performance, the angle of incidence has to be controlled within this limit.
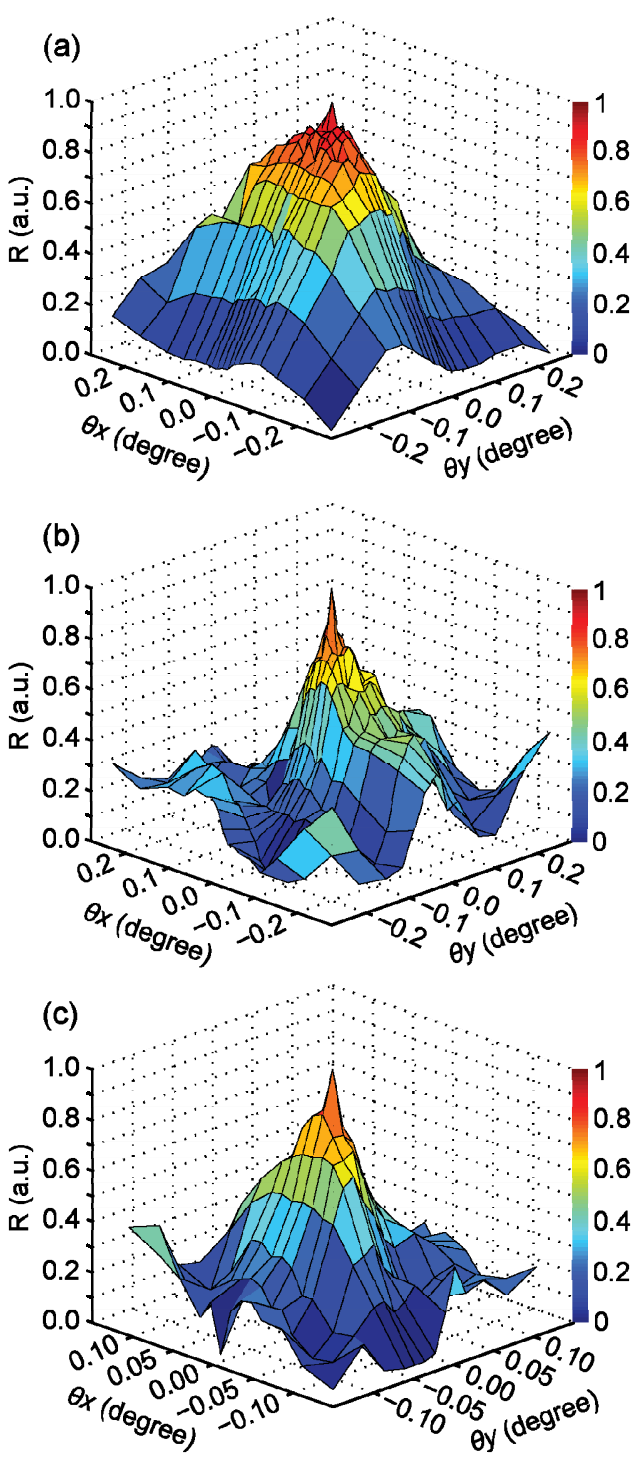

Fig. S15. Measured angular response $R(\theta x, \theta y)$ at $d=0.3 \mathrm{~mm}(\mathrm{a}), 0.5 \mathrm{~mm}$ (b) and $1.5 \mathrm{~mm}$ (c).

\subsection{Noise}

Alignment errors, electronic noises and computational errors all contribute to the discrepancies between the reconstructed color (or spectrum) and the true color (or spectrum) values. Therefore, it is important to understand how noises present in the gray-scale raw image deteriorate the reconstruction outcomes. By numerically adding Gaussian noises with various standard deviations to the gray-scale raw images, Fig. S16 summarizes the color errors (in percentage) versus the calculated signal-to-noise ratio (SNR) for different spectra. The spectra are randomly generated and the raw images are numerically synthesized by equation $\mathbf{S}=\mathbf{P S F} \cdot \boldsymbol{\Psi}$ with the knowledge of the calibrated SSPSF. According to the $10 \%$ color error tolerance (see Fig. S11 and Fig. S14), they estimate a SNR threshold of $20 \mathrm{~dB} \pm 3 \mathrm{~dB}$. 

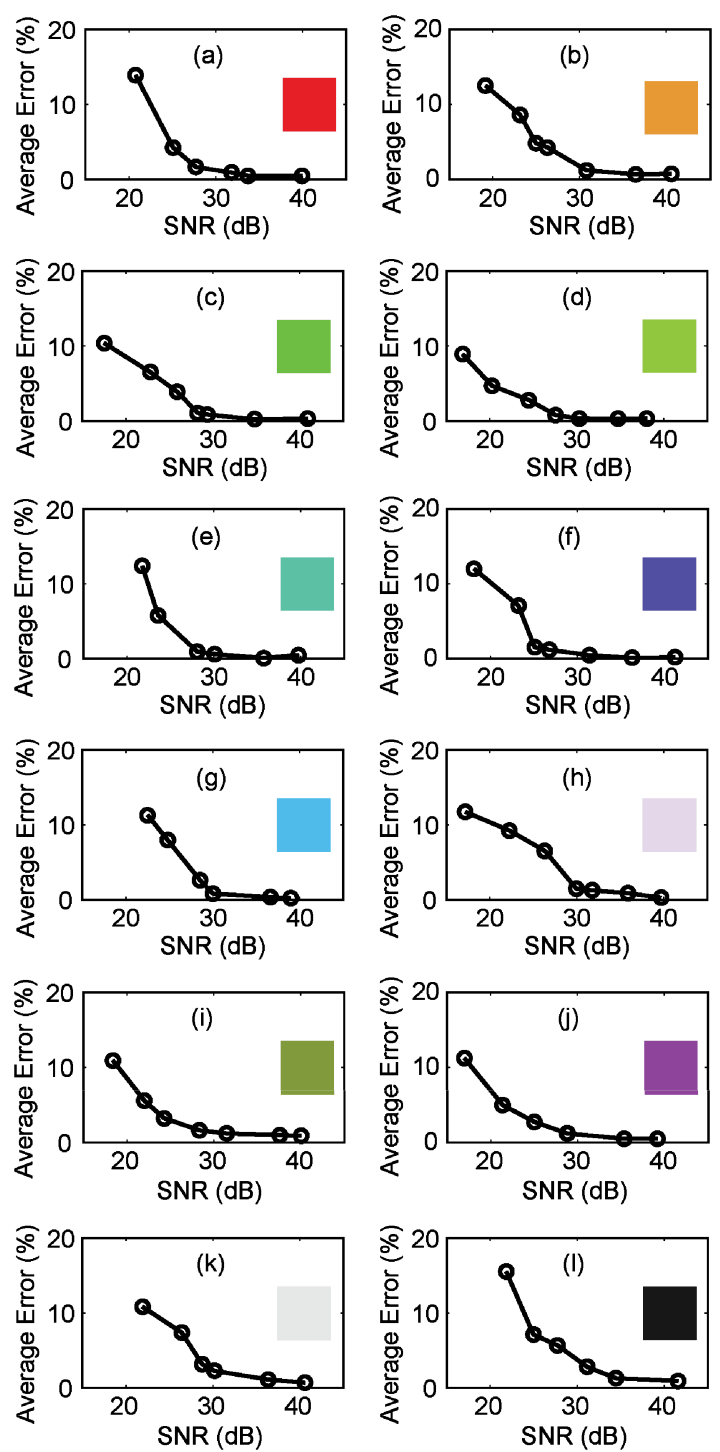

Fig. S16. Color reconstruction error (in percentage) averaged over R, G and B channels versus various SNR levels for different computergenerated random spectra. The color of different spectra are shown as insets.

\section{Raw images in image reconstruction experiment}

The raw images in experimental reconstructions of the rainbow object are summarized in Fig. S17. Note that the noises present in the reconstruction results (Figs. S17(a) and (b)) are largely suppressed by the simple de-noising technique, as shown in Figs. 4(c) and (d) in the main text. The filter to de-noise the hyperspectral maps (Figs. S17(c) and (d)) is exactly the same with the filter used to de-noise the RGB images (Figs. S17(a), (b) and Figs. $4(\mathrm{c})$ and $(\mathrm{d})$ ).
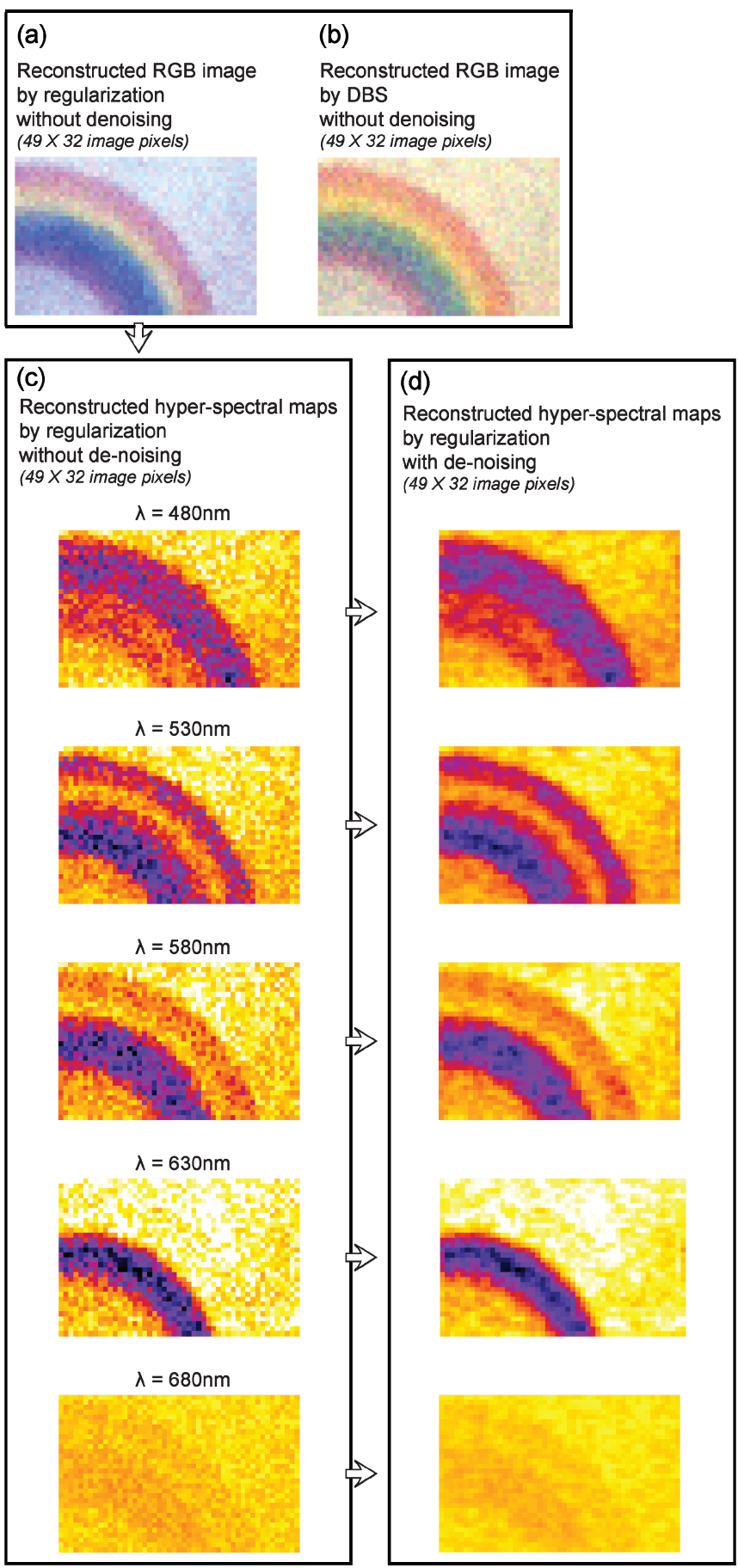

Fig. S17. Raw reconstructed images for the rainbow object by regularization (a) and DBS algorithm (b). They are before applying any de-noising process. Reconstructed hyper-spectral images at five wavelengths (a) before applying any de-noising process and (b) after applying a simple de-noising technique.

\section{Numerical simulation for smaller sensor pixel}

\subsection{Pseudo spectra of different colors}

In order to build a ground true hyperspectral data cube, it is necessary to firstly synthesize spectra of different colors. Since all colors can be broken down into three elementary colors: blue, green and red, they can be expressed as linear combinations of the spectra of these three elementary colors. Figures S18(a) - (c) give the spectra of the three elementary colors assumed in this simulation. Nine wavelength samples from $430 \mathrm{~nm}$ to $670 \mathrm{~nm}$ with 
30nm spacing are used. Again, for regularization, the number of wavelengths has to be equal to the number of sensor pixels occupied by one DFA unit $(3 \times 3$, as shown in Fig. $6($ a) $)$. Figures S18(d) - (f) show the spectra of three color examples represented by the elementary spectra.
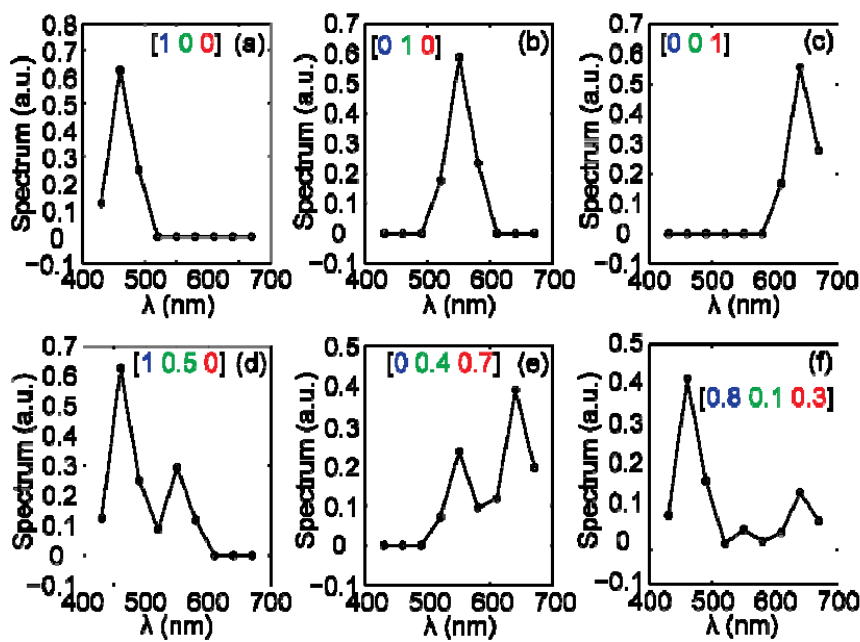

Fig. S18. Plots of the synthesized spectra. (a) - (c) Spectra of three elementary colors: blue, green and red. (d) - (f) Spectra of three example colors represented by the spectra of three elementary colors. The RGB color space is denoted by vectors in square brackets.

\subsection{Image synthesis and reconstruction}

To numerically synthesize the grayscale raw image, it is required to generate the light field diffracted by a single DFA unit cell. Here, finite-aperture boundary condition is used. Since we use coherent illumination, complex field is needed. Figure S19(a) summarizes the electric field ( $d=10 \mu \mathrm{m}$ gap) diffracted by a single DFA unit cell at nine discrete wavelengths. Therefore, the entire image of single wavelength received by the sensor array is just the intensity of the convolution of the ground true hyperspectral data cube with the simulated single-DFA-unit-cell diffraction pattern. Eventually, the final grayscale raw image is the summation of the images of all the contributed wavelengths.

$$
I(x, y)=\sum_{\lambda} I(x, y, \lambda)=\sum_{\lambda}\left|U\left(x^{\prime}, y^{\prime}, \lambda\right) \otimes H(x, y, \lambda)\right|^{2}
$$

Here, $I(x, y, \lambda)$ is the intensity of single wavelength, $U\left(x^{\prime}, y^{\prime}, \lambda\right)$ is the complex diffraction pattern of single wavelength (see Fig. S19(a)) simulated by the Fresnel formulations described by Eqs. (S1) - (S3), and $H(x, y, \lambda)$ is the hyperspectral data cube.

In addition, in Fig. $S 19(\mathrm{a})$, the green box represents a single DFA unit cell of $5 \mu \mathrm{m}$ by $5 \mu \mathrm{m}$ area. Its height profile is shown in Fig. 6(a) in the main text with 5 by 5 squares and $1 \mu \mathrm{m}$ square dimension. And the extent that the single DFA unit cell affects its neighbors is about $13 \mu \mathrm{m}$ by $13 \mu \mathrm{m}$. This essentially determines its spatial resolving power in contact imaging mode.

In order to follow exactly how experiment was carried out, for spectrum reconstruction, it is required to derive the diffraction patterns by periodic DFA units. This mimics uniform illumination in SS-PSF calibration. Again, using Eqs. (S1) - (S3), we simulate the diffraction patterns at nine wavelengths, plotted in Fig. S19(b). The actual sensor image is estimated by integrating light intensity delimited by the 3 by 3 uniform grid with sensor dimension of $1.67 \mu \mathrm{m}$ (white dashed lines in Fig. S19(b)).

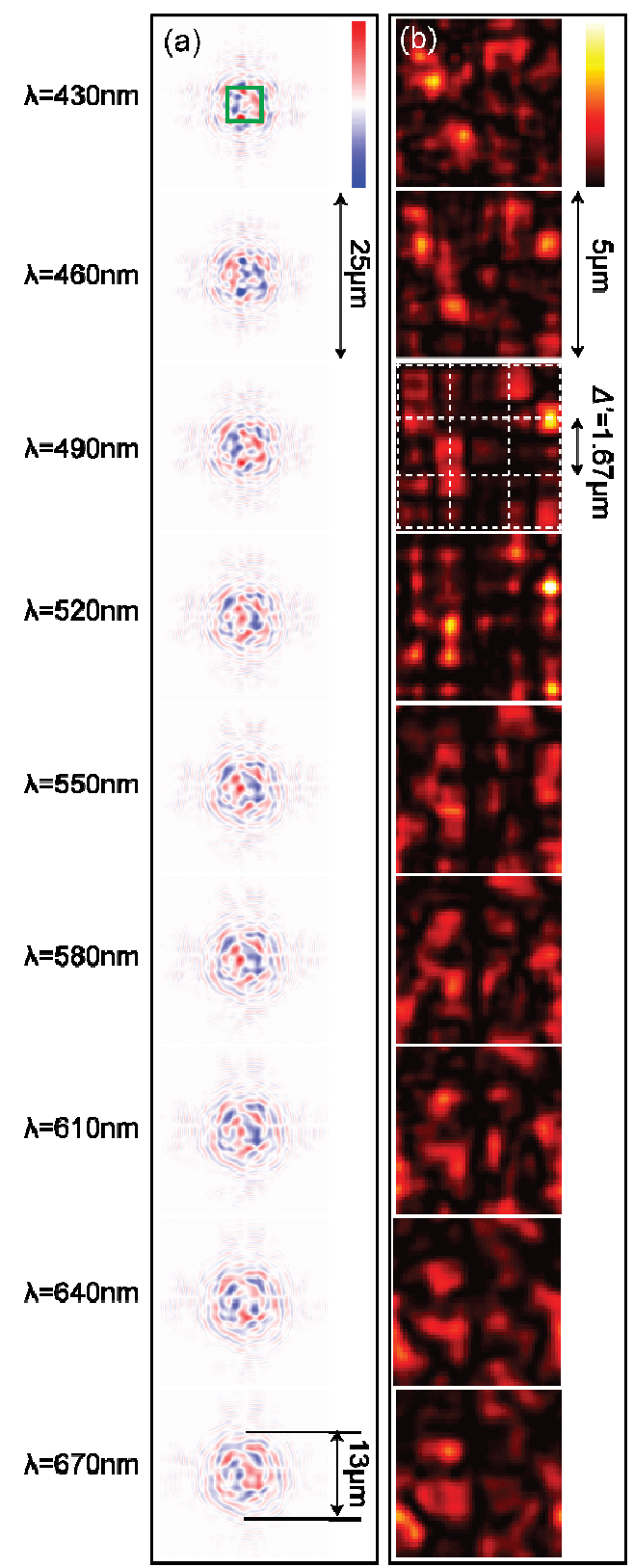

Fig. S19. (a) Simulated electric field diffracted by a single DFA unit cell at nine wavelengths from $430 \mathrm{~nm}$ to $670 \mathrm{~nm}$. The simulation area is $25 \mu \mathrm{m} \times 25 \mu \mathrm{m}$. The green block stands for single DFA unit and its diffraction pattern affects an area of $13 \mu \mathrm{m} \times 13 \mu \mathrm{m}$ at gap $d=10 \mu \mathrm{m}$ and $\lambda=670 \mathrm{~nm}$. (b) Simulated diffraction patterns by periodic DFA at nine wavelengths. The intensity is normalized. The gap is also $d=10 \mu \mathrm{m}$. Each unit cell has an area of $5 \mu \mathrm{m} \times 5 \mu \mathrm{m}$, occupying $3 \times 3$ sensor pixels with pixel dimension of $1.67 \mu \mathrm{m}$. Finite-aperture boundary condition is assumed in (a), while periodic boundary condition is assumed in (b).

\subsection{Reconstruction errors}

As discussed in the main text and section 8 of this Supplementary Materials, the cross-talk effect reduces the spatial resolution and affects color reconstruction accuracy, therefore it is necessary to briefly quantify this effect. Figs. S20(a)-(c) give the absolute error between the reconstruction and the original true images in B, G and R three color channels. The absolution errors averaged over the entire 2D image are 9,15 and 13 (in terms of 8bit intensity levels), respectively. The absolute error map averaged over the RGB three channels is shown in Fig. S20(d). 

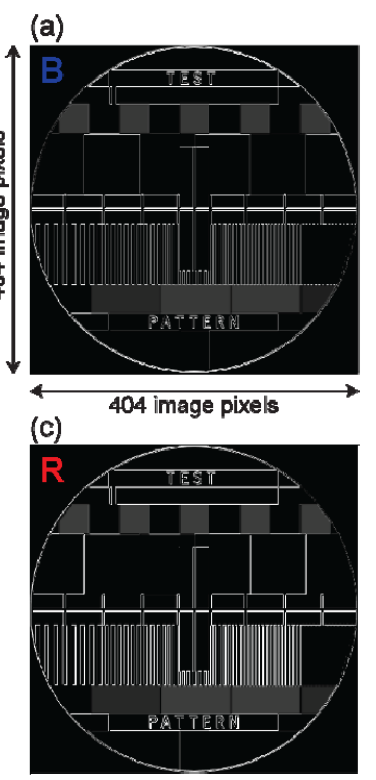

(b)

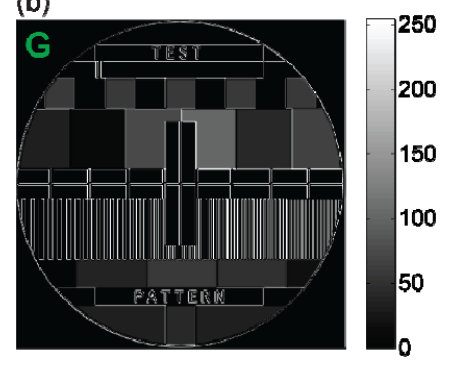

(d)

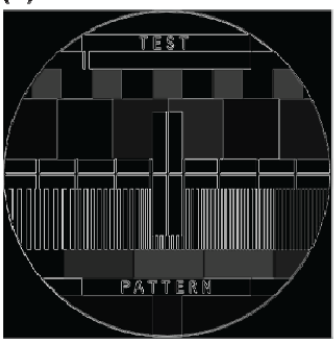

Fig. S20. Absolute error maps of color reconstruction shown in Fig. 6(d) for B (a), G (b) and R (c) color channels. They are quantified in terms of 8-bit intensity levels. (d) The absolute error map averaged over the three color channels in (a) - (c).

\section{References}

1. J. W. Goodman, Introduction to Fourier Optics. (Roberts and Company Publishers, 2005).

2. G. Kim, J. A. Dominguez-Caballero, and R. Menon, "Design and analysis of multi-wavelength diffractive optics," Opt. Express 20, 2814-2823 (2012).

3. K. Reimer, H. J. Quenzer, M. Jurss, and B. Wagner, "Micro-optic fabrication using one-level gray-tone lithography," Proc. SPIE 3008, 279288 (1997).

4. Data sheet of Heidelberg $\mu$ PG 101: http://www.himt.de/en/products/muepg101.php

5. Data sheet of Shipley 1813: http://www.nanophys.kth.se/nanophys/facilities/nfl/resists/S1813/s18 00seriesDataSheet.pdf

6. Data sheets of high-precision optical mounts from Thorlabs: http://www.thorlabs.us/thorproduct.cfm?partnumber=PRM2 http://www.thorlabs.us/thorproduct.cfm?partnumber=CXY2

7. P. Wang, J. A. Dominguez-Caballero, D. J. Friedman, and R. Menon, "A new class of multi-bandgap high-efficiency photovoltaics enabled by broadband diffractive optics," Prog. Photovolt: Res. Appl. 23(9), 10731079 (2015).

8. P. Wang and R. Menon, "Optimization of generalized dielectric nanostructures for enhanced light trapping in thin-film photovoltaics via boosting the local density of optical states," Opt. Exp. 22(51), A99A110 (2014).

9. P. Wang and R. Menon, "Optimization of periodic nanostructures for enhanced light-trapping in ultra-thin photovoltaics," Opt. Exp. 21(5), 6274-6285 (2013).

10. P. Wang and R. Menon, "Optical microlithography on oblique and multiplane surfaces using diffractive phase masks," J. Micro/Nanolith. MEMS MOEMS 14(2), 023507 (2015).

11. P. Wang and R. Menon, "Computational spectrometer based on a broadband diffractive optic," Opt. Exp. 22(12), 14575-14587 (2014).

12. P. Wang and R. Menon, "Computational spectroscopy via singularvalue-decomposition and regularization," Opt. Exp. 22(18), 2154121550 (2014).

13. P. C. Hansen, Discrete Inverse Problems: Insight and Algorithms (SIAM Press, 2010). 